\title{
Padé-Improved Estimates of Hadronic Higgs Decay Rates
}

\author{
V. Elias and F. A. Chishtie \\ Department of Applied Mathematics \\ The University of Western Ontario \\ London, Ontario N6A 5B7 CANADA \\ T. G. Steele \\ Department of Physics and Engineering Physics \\ University of Saskatchewan \\ Saskatoon, Saskatchewan S7N 5E2 CANADA
}

November 10, 2018

\begin{abstract}
Asymptotic Padé-approximant methods are utilized to estimate the $\mathcal{O}\left(\alpha_{s}^{5}\right)$ contribution to the $H \rightarrow g g$ rate and the $\mathcal{O}\left(\alpha_{s}^{4}\right)$ contribution to the $H \rightarrow b \bar{b}$ rate. The former process is of particular interest because of the slow convergence evident from the three known terms of its QCD series, which begins with an $\mathcal{O}\left(\alpha_{s}^{2}\right)$ leading-order term. The $\mathcal{O}\left(\alpha_{s}^{5}\right)$ contribution to the $H \rightarrow g g$ rate is expressed as a degree-3 polynomial in $L \equiv \ln \left(\mu^{2} / m_{t}^{2}(\mu)\right)$. We find that asymptotic Padé-approximant predictions for the coefficients of $L, L^{2}$, and $L^{3}$ are respectively within $1 \%, 2 \%$, and $7 \%$ of true values extracted via renormalization-group methods. Upon including the full set of next-order coefficients, the $H \rightarrow g g$ rate is found to be virtually scale-independent over the $0.3 M_{H} \lesssim \mu \lesssim M_{t}$ range of the renormalization scale-parameter $\mu$. We conclude by discussing the small $\mathcal{O}\left(\alpha_{s}^{4}\right)$ contribution to the $H \rightarrow b \bar{b}$ rate, which is obtained from a prior asymptotic Padé-approximant estimate of the $\mathcal{O}\left(\alpha_{s}^{4}\right)$ contribution to the quark-antiquark scalar-current correlation function.
\end{abstract}

\section{Introduction}

The Higgs boson characterizes the electroweak symmetry breaking underlying the Standard Model. Its discovery and phenomenology will be of immense importance in clarifying our understanding of this symmetry breaking, as well as in providing vital information as to the nature of beyond-the-Standard-Model physics. The two leading hadronic decay modes of a Weinberg-Salam Higgs boson $(H)$ with mass between $100 \mathrm{GeV}$ and $160 \mathrm{GeV}$ are the QCD processes $H \rightarrow b \bar{b}$ and $H \rightarrow$ two gluons $(g g)$. Although the rate for this latter process is known to $\mathcal{O}\left(\alpha_{s}^{4}\right)$, such precision incorporates only two non-leading orders of a slowly converging series in the strong coupling. If $M_{H}=100 \mathrm{GeV}$, for example, the known order-by-order QCD corrections have been calculated [1] to be $1+0.66+0.21$.

The work presented here is primarily directed toward obtaining a more precise estimate of the $H \rightarrow g g$ decay rate. We utilize renormalization-group $(R G)$ and asymptotic Padé-approximant methods to estimate the full nextorder contribution to the underlying correlation function for this process. Such an approach has already been applied to the quark-antiquark scalar-current correlation function underlying the $H \rightarrow b \bar{b}$ rate [2], a calculation we review in Section 5 of the present paper.

As in our prior analysis of the two-gluon decay amplitude of a non-Standard-Model CP-odd Higgs field [3], the approach we take here is to test asymptotic Padé-approximant estimates against RG-accessible coefficients within the next-order of perturbation theory. We show such estimates to be accurate up to relative errors of only a few percent, supporting the credibility of the same approach in estimating the RG-inaccessible coefficient needed to determine the full $\mathcal{O}\left(\alpha_{s}^{5}\right)$ contribution to the $H \rightarrow g g$ rate. 
We operate within the context of $\overline{M S}$ expressions for the $H \rightarrow g g$ and $H \rightarrow b \bar{b}$ rates that explicitly depend on an arbitrary renormalization scale $\mu$. It has been argued elsewhere 4,0 , 6 that asymptotic Padé-approximant methods reduce the explicit scale-dependence of perturbative quantities which must ultimately be scale invariant. We find this to be the case for the $H \rightarrow g g$ rate as well, despite residual scale-sensitivity anticipated from the estimated RG-inaccessible coefficient of the nonlogarithmic $\mathcal{O}\left(\alpha_{s}^{5}\right)$ term within the next-order correlator.

In Section 2, we demonstrate the explicit RG-invariance of the $H \rightarrow g g$ rate, as calculated in $[1]$ in the $m_{b} \rightarrow 0, M_{H}^{2}<<4 M_{t}^{2}$ limit. This RG invariance enables one to calculate all the next-order coefficients $c_{k}$ of $l n^{k}\left[\mu^{2} / m_{t}\left(\mu^{2}\right)\right]\left(\alpha_{s}(\mu) / \pi\right)^{5}$ within the calculated rate; only the $k=0$ constant term is RG-inaccessible.

In Section 3, we review how asymptotic Padé-approximant methods may be utilized to estimate this set of next-order coefficients, and demonstrate close agreement with the RG-determinations of Section 2 over the range $100 \leq M_{H} \leq 175 \mathrm{GeV}$. The RG-inaccessible coefficient $c_{0}$ is also estimated over this range of Higgs masses and is fitted to its anticipated behaviour as a degree-3 polynomial in $\ln \left[\left(M_{H}^{2} / M_{t}^{2}\right)_{\text {pole }}\right]$.

In Section 4 we examine the residual scale dependence of the Padé-improved $H \rightarrow g g$ decay rate, as obtained in Section 3. Although we anticipate a relative scale dependence comparable to $c_{0}\left(\alpha_{s}(\mu) / \pi\right)^{3}(\approx 3-10 \%)$, since $c_{0}$ cannot be extracted perturbatively from the RG equation (to the order we consider), we find that the residual scale dependence of the decay rate is a full order of magnitude smaller than this estimate for $\mu$ between $0.3 M_{H}$ and $M_{t}$ (we assume $100 \mathrm{GeV} \leq M_{H} \leq 175 \mathrm{GeV}$ ). Consequently, the scale-dependence of the Padé-derived term $c_{0}\left(\alpha_{s}(\mu) / \pi\right)^{5}$ very nearly cancels the scale-dependence anticipated from truncation of the perturbative series for the $H \rightarrow g g$ rate, thereby facilitating the (nearly) scale-invariant rate predictions of Table 6 . Predictions of the $H \rightarrow g g$ decay rate are tabulated both for the $m_{b} \rightarrow 0, M_{H}^{2}<<4 M_{t}^{2}$ limit, and for the leading-order departures from this limit for appropriate constant and running values for $m_{b}$ and $M_{t}$.

In the concluding section, we review how prior asymptotic Padé-approximant estimates of the $\mathcal{O}\left(\alpha_{s}^{4}\right)$ contribution to the scalar-current correlation function [2] can be incorporated into the $H \rightarrow b \bar{b}$ decay rate. We demonstrate that the resulting next-order contribution, though only $0.01 \%$ of the leading term, is somewhat larger than known $\mathcal{O}\left(\alpha_{s}^{2}\right)$ power-suppressed contributions [7]. Consequently, the estimated $\mathcal{O}\left(\alpha_{s}^{4}\right)$ term enables the $H \rightarrow b \bar{b}$ rate to be estimated to four significant figure accuracy.

\section{RG-Invariance of the $H \rightarrow g g$ Rate}

The Higgs $\rightarrow$ gg decay rate is given explicitly to 3-loop order in [1] by the following expressions:

$$
\begin{aligned}
& \Gamma_{H \rightarrow g g}=\frac{\sqrt{2} G_{F}}{M_{H}} R\left(\alpha_{s}, q^{2}=M_{H}^{2}, \mu^{2}, M_{t}^{2}\right), \\
& R=C_{1}^{2} \operatorname{Im}<\left[0_{1}^{\prime}\right]^{2}>, \\
& C_{1}=-\frac{x^{(6)}}{12}\left[1+x^{(6)}\left(\frac{11}{4}-\frac{1}{6} \ln \left(\frac{\mu^{2}}{M_{t}^{2}}\right)\right)\right. \\
& \left.+\left(x^{(6)}\right)^{2}\left(\frac{211}{36}+\frac{55}{48} \ln \left(\frac{\mu^{2}}{M_{t}^{2}}\right)+\frac{1}{36} \ln ^{2}\left(\frac{\mu^{2}}{M_{t}^{2}}\right)\right)+\mathcal{O}\left[\left(x^{(6)}\right)^{3}\right]\right] \\
& \text { Im }<\left[0_{1}^{\prime}\right]^{2}>=\frac{2 q^{4}}{\pi}\left[1+x^{(5)}\left(\frac{149}{12}+\frac{23}{6} \ln \left(\frac{\mu^{2}}{q^{2}}\right)\right)\right. \\
& \left.+\quad\left(x^{(5)}\right)^{2}\left(68.64817+\frac{1297}{16} \ln \left(\frac{\mu^{2}}{q^{2}}\right)+\frac{529}{48} \ln ^{2}\left(\frac{\mu^{2}}{q^{2}}\right)\right)+\mathcal{O}\left[\left(x^{(5)}\right)^{3}\right]\right] .
\end{aligned}
$$

In the above expression, $<\left[0_{1}^{\prime}\right]^{2}>$ is the vacuum polarization of the Higgs field induced via the gluon operator $0_{1}^{\prime}=G_{a \mu \nu} G_{a}^{\mu \nu}$ [1] , and $x^{\left(n_{f}\right)} \equiv \alpha_{s}^{\left(n_{f}\right)} / \pi$, where $\alpha_{s}^{\left(n_{f}\right)}(\mu)$ is the running strong coupling with $n_{f}$ active flavours. 
Five flavours are assumed to be light in both (2.1c) and (2.1d). The $t$-quark mass $M_{t}$ appearing in (2.1c) is an RG-invariant pole mass, and $M_{H}^{2}$ is assumed to be small compared to $4 M_{t}^{2}$ [1].

Our goal here is to use asymptotic Padé-approximant methods in conjunction with the RG-invariance of (2.1a) in order to predict the next order contribution to $\Gamma(H \rightarrow g g)$. In a previous application [6] of such methods to the inclusive semileptonic $b \rightarrow u$ rate, it was found that success in predicting RG-accessible next-order coefficients was greatly enhanced by recasting the entire expression in terms of the running fermion mass. Indeed, such replacement of the $b$-quark pole mass with its scale-dependent $\overline{M S}$ mass had already been employed by van Ritbergen [8] to avoid a renormalon pole.

Consequently, we first re-express the $H \rightarrow 2 g$ rate in terms of the running $t$-quark mass $m_{t}(\mu)$, which evolves via a six-active-flavour $\gamma_{m}$-function, and the corresponding six-active-flavour running coupling $x^{(6)}(\mu)$. This transformation is facilitated by the following relationships [9]:

$$
\begin{aligned}
& x^{(5)}(\mu)=x^{(6)}(\mu)-\frac{\left(x^{(6)}(\mu)\right)^{2}}{6} \ln \left(\frac{\mu^{2}}{m_{t}^{2}(\mu)}\right)+\mathcal{O}\left[\left(x^{(6)}\right)^{3}\right], \\
& m_{t}(\mu) / M_{t}=\left[1-x^{(6)}(\mu)\left(\frac{4}{3}+\ln \left(\frac{\mu^{2}}{M_{t}^{2}}\right)\right)+\mathcal{O}\left[\left(x^{(6)}\right)^{2}\right] .\right.
\end{aligned}
$$

Equation (2.3) implies that the logarithm in (2.1c) may be re-expressed in terms of $L \equiv \ln \left(\mu^{2} / m_{t}^{2}(\mu)\right)$ :

$$
\ln \left(\frac{\mu^{2}}{M_{t}^{2}}\right)=L-x^{(6)}(\mu)[2 L+8 / 3]+\mathcal{O}\left[\left(x^{(6)}\right)^{2}\right]
$$

With $q^{2}=M_{H}^{2}$, the logarithm in (2.1d) can also be expressed entirely in terms of the running $t$-quark mass and a $\operatorname{logarithm} T \equiv \ln \left(M_{H}^{2} / M_{t}^{2}\right)$ of the ratio of $R G$-invariant pole masses:

$$
\ln \left(\frac{\mu^{2}}{M_{H}^{2}}\right)=L-T-x^{(6)}(\mu)[2 L+8 / 3]+\mathcal{O}\left[\left(x^{(6)}\right)^{2}\right] .
$$

Substitution of (2.2) and (2.5) into (2.1d) with $q^{2}=M_{H}^{2}$, and substitution of (2.4) into (2.1c) leads to the following expression for the $H \rightarrow g g$ decay rate:

$$
\begin{aligned}
\Gamma_{H \rightarrow g g}=\frac{\sqrt{2} G_{F} M_{H}^{3}}{72 \pi} S\left[x^{(6)}(\mu), L(\mu), T\right] \\
\begin{aligned}
S[x, L, T]=x^{2}(1+ & x\left[\left(\frac{215}{12}-\frac{23 T}{6}\right)+\frac{7}{2} L\right] \\
& +x^{2}\left[\left(146.8912-\frac{4903}{48} T+\frac{529}{48} T^{2}\right)+\left(\frac{1445}{16}-\frac{161}{8} T\right) L+\frac{147}{16} L^{2}\right] \\
& \left.+x^{3}\left[c_{0}+c_{1} L+c_{2} L^{2}+c_{3} L^{3}\right]+\mathcal{O}\left(x^{4}\right)\right) .
\end{aligned}
\end{aligned}
$$

In (2.7), we list the unknown coefficients $c_{0}, c_{1}, c_{2}$ and $c_{3}$ of the 4-loop contribution to the rate. Three of these may be extracted by the scale-[RG-] invariance of the physical decay rate: $d \Gamma / d \mu=0$. This invariance implies that

$$
\begin{array}{r}
O=\mu \frac{d S}{d \mu}[x, L, T], \\
=\left[1-2 \gamma_{m}(x)\right] \frac{\partial S}{\partial L}+\beta(x) \frac{\partial S}{\partial x} .
\end{array}
$$

Both the $\beta$ and $\gamma_{m}$ functions in (2.8) are referenced to six active flavours:

$$
\beta^{(6)}(x)=-\frac{7}{4} x^{2}-\frac{13}{8} x^{3}+\frac{65}{128} x^{4} \ldots
$$




$$
\gamma_{m}^{(6)}(x)=-x-\frac{27}{8} x^{2} \ldots
$$

One can easily verify from the known terms listed in (2.7) that (2.8) is perturbatively valid to orders $x^{3}, x^{4}$ (including explicit cancellation of terms involving $T$ ), and $x^{4} L$. The continued perturbative validity of (2.8) to orders $x^{5} L^{2}$, $x^{5} L$, and $x^{5}$ is sufficient to determine the four-loop coefficients $c_{1}, c_{2}$, and $c_{3}$ :

$$
\begin{aligned}
& c_{1}=910.3167-\frac{16643}{24} T+\frac{3703}{48} T^{2}, \\
& c_{2}=\frac{1225}{4}-\frac{1127}{16} T, \\
& c_{3}=\frac{343}{16} .
\end{aligned}
$$

The coefficient $c_{0}$ is not RG-accessible to these orders. In the next section, we will utilize asymptotic Padé approximant methods to estimate the four-loop coefficients $\left\{c_{0}, c_{1}, c_{2}, c_{3}\right\}$. As in prior work [2, 3, 6], the accuracy of these predictions in reproducing $(2.11-2.13)$ will serve as an indication of the accuracy of our estimate for $c_{0}$.

\section{Padé-Predictions for the Four-Loop Coefficients}

The asymptotic Padé-approximant procedure for estimation of the four loop coefficients $c_{0}, c_{1}, c_{2}, c_{3}$ in $(2.7)$ has been delineated in previous work [2, 33, 6]. The series (2.7) may be expressed in the form

$$
S[x, L, T]=x^{2}\left[1+R_{1}[L, T] x+R_{2}[L, T] x^{2}+R_{3}[L, T] x^{3}+\ldots\right]
$$

where $R_{1}$ and $R_{2}$ are known, and $R_{3}$ is to be determined:

$$
\begin{aligned}
& R_{1}[L, T]=\left(\frac{215}{12}-\frac{23}{6} T\right)+\frac{7}{2} L \\
& R_{2}[L, T]=146.8912-\frac{4903}{48} T+\frac{529}{48} T^{2}+\left(\frac{1445}{16}-\frac{161}{8} T\right) L+\frac{147}{16} L^{2} \\
& R_{3}[L, T]=c_{0}(T)+c_{1}(T) L+c_{2}(T) L^{2}+c_{3} L^{3} .
\end{aligned}
$$

Initially, we shall eliminate $T$ as a variable by assuming that the Higgs pole mass is $100 \mathrm{GeV}$, in which case $T=\ln \left[M_{H}^{2} / M_{t}^{2}\right]=2 \ln (100 / 175.6)=-1.126$. As described in [6] and [10, the [0|1] Padé-approximant prediction for $R_{2}$ is

$$
R_{2}^{[0 \mid 1]}=R_{1}^{2}
$$

and the [1|1] Padé-approximant prediction for $R_{3}$ is

$$
R_{3}^{[1 \mid 1]}=R_{2}^{2} / R_{1} .
$$

If the error of $[N \mid 1]$ approximants in predicting $R_{N+2}$, the $N+2$ term in the perturbative series, is inversely proportional to $N+1$ - i.e., if

$$
\frac{R_{N+2}^{[N \mid 1]}-R_{N+2}^{\text {exact }}}{R_{N+2}^{\text {exact }}}=\frac{-A}{N+1},
$$


where $A$ is a constant - one may then utilize (3.5) and the exact value for $R_{2}$ within (3.7) to obtain $A=\left(R_{2}^{2}-\right.$ $\left.R_{1}^{2}\right) / R_{2}$. Substituting (3.6) and this estimate for $A$ into (3.7), one obtains an error-improved estimate for the unknown coefficient $R_{3}$ [11]:

$$
R_{3}[L]=2 R_{2}^{3}[L] /\left(R_{1}[L] R_{2}[L]+R_{1}^{3}[L]\right)
$$

To obtain estimates of the coefficients $c_{0}, c_{1}, c_{2}, c_{3}$ within (3.4), we match the scale dependence of (3.4) to that of (3.8) over the purely perturbative $L>0$ region [corresponding to the ultraviolet scales $\mu>m_{t}(\mu)$ ] through use of the moment integrals

$$
N_{k} \equiv(k+2) \int_{0}^{1} d w w^{k+1} R_{3}(w)
$$

where $w=m_{t}^{2}(\mu) / \mu^{2}[L=-\ln (w)]$. Substitution of (3.4) into the integrand of (3.9) yields the following expressions for the first four moments [2]:

$$
\begin{aligned}
& N_{-1}=c_{0}+c_{1}+2 c_{2}+6 c_{3}, \\
& N_{0}=c_{0}+\frac{1}{2} c_{1}+\frac{1}{2} c_{2}+\frac{3}{4} c_{3}, \\
& N_{1}=c_{0}+\frac{1}{3} c_{1}+\frac{2}{9} c_{2}+\frac{2}{9} c_{3}, \\
& N_{2}=c_{0}+\frac{1}{4} c_{1}+\frac{1}{8} c_{2}+\frac{3}{32} c_{3} .
\end{aligned}
$$

However, explicit numerical estimates of these four moments may be obtained via substitution of (3.2) and (3.3) for the respective factors of $R_{1}[L]$ and $R_{2}[L]$ appearing in (3.8), and by subsequent substitution of this estimate for $R_{3}[L]$ into the integrand of (3.9) [with $L=-\ln (w)$ ]. For $M_{H}=100 \mathrm{GeV}$ the resulting estimates are

$$
N_{-1}=5102.9, N_{0}=3542.9, N_{1}=3131.8, N_{2}=2944.7 \text {. }
$$

Substitution of these values into (3.10) yields the following predicted values $\left(c_{i}^{\text {Padé }}\right)$ for the four-loop terms in the $H \rightarrow g g$ decay rate $(2.7)$ :

$$
c_{0}^{\text {Padé }}=2453, c_{1}^{\text {Padé }}=1772, c_{2}^{\text {Padé }}=378.4, c_{3}^{\text {Padé }}=20.27 .
$$

The exact values of $c_{1}, c_{2}$, and $c_{3}$ were determined via $R G$ methods in the previous section. For $M_{H}=100 \mathrm{GeV}$ [i.e. for $T=-1.126]$, these values are found from (2.11-13) to be

$$
c_{1}=1789.02, c_{2}=385.568, c_{3}=21.4375
$$

Comparing (3.12) and (3.13), one finds the relative error $\left[\delta c_{i} \equiv\left(c_{i}^{\text {Padé }}-c_{i}\right) / c_{i}\right]$ of the Padé estimates for $c_{1}, c_{2}$ and $c_{3}$ to be $-0.95 \%,-1.9 \%$, and $-5.5 \%$, respectively.

Such accuracy in predicting the known four-loop terms in the $H \rightarrow g g$ rate suggests that the prediction for $c_{0}$ in (3.12) is a credible one. One way to test the stability of this prediction is to utilize the true values of $c_{1}, c_{2}, c_{3}$ within the moment expressions (3.10) to obtain [for the numerical values (3.11) already obtained for these moments] four independent determinations of $c_{0}$. We then find that

$$
\begin{array}{ll}
(3.10 a): & c_{0}=2412, \\
(3.10 b): & c_{0}=2436, \\
(3.10 c): & c_{0}=2444, \\
(3.10 d): & c_{0}=2447,
\end{array}
$$


results all within $2 \%$ of that in (3.12).

An alternative approach to matching the four-loop coefficients $c_{i}$ within (3.4) to the error improved Padé estimate (3.8) is optimize the least-squares function [3]

$$
\chi^{2}\left[c_{0}, c_{1}, c_{2}, c_{3}\right]=\int_{0}^{1}\left[R_{3}-\left(c_{0}-c_{1} \ln w+c_{2} \ln ^{2} w-c_{3} \ln ^{3} w\right)\right]^{2} d w,
$$

with $R_{3}$ in the integral given by (3.8). As before, factors of $R_{1}$ and $R_{2}$ appearing in (3.8) are given explicitly by (3.2) and (3.3) with $L=-\ln (w)$. One then finds for $M_{H}=100 \mathrm{GeV}[T=-1.126]$ that

$$
\begin{aligned}
\chi^{2}\left(c_{0}, c_{1}, c_{2}, c_{3}\right) & =4.064168878 \cdot 10^{7}+720 c_{3}^{2}+4 c_{0} c_{2}+c_{0}^{2}+2 c_{0} c_{1}+24 c_{2}^{2}+12 c_{1} c_{2}+12 c_{0} c_{3}+2 c_{1}^{2} \\
& +240 c_{2} c_{3}+48 c_{1} c_{3}-10205.87 c_{0}-17507.05 c_{1}-54106.23 c_{2}-234536.67 c_{3}
\end{aligned}
$$

The optimization requirement

$$
\frac{\partial \chi^{2}}{\partial c_{i}}=0
$$

yields predictions remarkably close to those of (3.12),

$$
c_{0}^{\chi^{2}}=2452, \quad c_{1}^{\chi^{2}}=1774, \quad c_{2}^{\chi^{2}}=377.2, \quad c_{3}^{\chi^{2}}=20.45,
$$

further confirming the stability of the estimation procedure.

In Table 1, we have tabulated a set of predictions of the four-loop term $c_{0}$ for values of the Higgs mass between 100 and $175 \mathrm{GeV}$. Also tabulated are the errors in the predicted values for $c_{1}, c_{2}, c_{3}$, relative to the true values for these coefficients, as given in (2.11-13). Estimated values of $c_{1}$ and $c_{2}$ remain within $2 \%$ of their true values (2.11-13) over the range of Higgs masses given; the relative error of $c_{3}$ estimates remains below $7 \%$ for the same range. This consistency provides further support for the $c_{0}$ estimates presented in the final column.

These $c_{0}$ estimates may be utilized to ascertain the convergence of the series (2.7). If we choose $\mu=m_{t}(\mu)$, the logarithmic factors $L^{k}$ within (2.7) all vanish. To evaluate the series $S / x^{2}$ in (2.7), we assume that $m_{t}\left(m_{t}\right) \cong$ $M_{t}=175.6 \mathrm{GeV}$ and that $\alpha_{s}(175.6)=0.10915=\pi x(175.6)$, as evolved from $\alpha_{s}\left(M_{z}\right)=0.119$ [12], in which case

$$
S(\mu=175.6 \mathrm{GeV}) / x^{2}(175.6)=1+a_{0} x(175.6)+b_{0} x^{2}(175.6)+c_{0} x^{3}(175.6) .
$$

For Higgs masses between 100-175 GeV, we tabulate in Table 2 the magnitudes of successive orders in (3.19). The final $4^{\text {th }}$ order term is obtained from the appropriate Padé estimate for $c_{0}$ listed in Table 1 . Table 2 shows that the four-loop term decreases from $10 \%$ to $3 \%$ of the leading contribution as $M_{H}$ increases from 100 to $175 \mathrm{GeV}$. Moreover, the convergence of the series is problematical in the absence of the estimate for the four loop term. Over the range of Higgs masses considered, the three-loop contribution is between $18 \%$ and $33 \%$ of the leading (one-loop) contribution.

The procedures delineated above can also be utilized to predict $c_{0}$ 's explicit polynomial dependence of $T$,

$$
c_{0}(T)=a_{0}+a_{1} T+a_{2} T^{2}+a_{3} T^{3},
$$

analogous to the expressions (2.11-13) obtained from $R G$-invariance for $c_{1}(T), c_{2}(T)$, and $c_{3}(T)$. To extract the $T$ dependence of $c_{0}$, we first incorporate all known $T$-dependence into the least- squares function (3.15):

$$
\chi^{2}\left[c_{0}(T)\right]=\int_{0}^{1}\left[R_{3}[L, T]-\left\{c_{0}(T)+c_{1}(T) L+c_{2}(T) L^{2}+c_{3}(T) L^{3}\right\}\right]^{2} d w .
$$

In (3.21), $L \equiv-\operatorname{lnw}$. The quantities $c_{1}(T), c_{2}(T)$ and $c_{3}(T)$ are no longer optimizable variables as in (3.15), but are now the explicit polynomials (2.11-13) obtained in the previous section via $R G$-methods. The factor $R_{3}[L, T]$ in the integrand of (3.21) is just (3.8) generalized to include the explicit $T$-dependence of $R_{1}$ and $R_{2}$ [eqs. (3.2) and (3.3)]:

$$
R_{3}[L, T]=\frac{2 R_{2}^{3}[L, T]}{R_{1}[L, T] R_{2}[L, T]+R_{1}^{3}[L, T]} .
$$


From (3.21), the requirement $d \chi^{2} / d c_{0}=0$ generates $c_{0}$ as a function of $T$. Since $T \equiv \ln \left(M_{H}^{2} / M_{T}^{2}\right)$, we restrict our attention to the region $-1 \leq T \leq 0$ (i.e. to values for $M_{H}$ between $M_{t}$ and $M_{t} e^{-1 / 2}=107 \mathrm{GeV}$ ). A set of values for $c_{0}(T)$ can be obtained via optimization of (3.21) over values of $T$ in this region:

$$
\begin{aligned}
c_{0}(0) & =735.7, c_{0}(-0.1)=841.7, c_{0}(-0.2)=955.6, c_{0}(-0.3)=1078, \\
c_{0}(-0.4) & =1208, c_{0}(-0.5)=1346, c_{0}(-0.6)=1493, c_{0}(-0.7)=1649, \\
c_{0}(-0.8) & =1814, ; c_{0}(-0.9)=1988, c_{0}(-1.0)=2170 .
\end{aligned}
$$

We obtain a least-squares fit of these results to the form (3.20) by optimizing

$$
\chi^{2}\left[a_{0}, a_{1}, a_{2}, a_{3}\right] \equiv \sum_{i=0}^{10}\left[c_{0}(-i / 10)-\left(a_{0}-\frac{a_{1} i}{10}+\frac{a_{2} i^{2}}{100}-\frac{a_{3} i^{3}}{1000}\right)\right]^{2}
$$

with respect to $\left\{a_{0}, a_{1}, a_{2}, a_{3}\right\}$, and find that

$$
c_{0}(T)=735.7-1020 T+388.8 T^{2}-25.41 T^{3} .
$$

Each coefficient listed above is within $5 \%$ relative error of the corresponding coefficient obtained via a least squares fit of the $c_{0}$ values displayed in Table 1 to the polynomial form (3.20):

$$
c_{0}(T)=755.9-1029 T+394.3 T^{2}-26.74 T^{3} .
$$

Note that (2.11), (2.12), (2.13) and the Padé-estimates (3.25) or (3.26) specify all the logarithmic coefficients within the full four loop contribution (3.4) to the $H \rightarrow g g$ decay rate (2.6,7), and comparison of these results with future perturbative calculations should yield information which can be employed to further improve Padé estimation procedures. The exact $H \rightarrow g g$ rate (2.6) is necessarily a scale invariant physical quantity. The factor $S[x(\mu), L(\mu), T]$ within (2.6) will exhibit residual scale-dependence [i.e. $\mu$-dependence] only as a consequence of truncation of the series $S$ to a given order of perturbation theory. In the section that follows, the incorporation of the four-loop coefficients $c_{0-3}$ of $S[x(\mu), L(\mu), T]$ is seen to eliminate virtually all of this residual scale dependence.

\section{The $H \rightarrow g g$ Rate}

In Fig. 1, the $\mu$-dependence of three- and four-loop expressions for $S\left[x^{(6)}(\mu), L(\mu), T\right]$ is plotted for the case of a $140 \mathrm{GeV}$ Higgs mass $[T=2 \ln (140 / 175.6)]$. The four-loop term within $S$ is evaluated through use of the Padé estimate $c_{0}=1306$ (Table 1 ) in conjunction with eqs. (2.11-2.13) for the $R G$-accessible coefficients $c_{1}, c_{2}$, and $c_{3}$. The running coupling $\alpha_{s}(\mu)$ and running mass $m_{t}(\mu)$ occurring within (2.7) are evolved via four-loop $\beta$ and $\gamma$-functions from physical reference values $\alpha_{s}\left(M_{z}\right)=0.119$ and $m_{t}\left(m_{t}\right)=175.6 \mathrm{GeV}$ [12].

Figure 1 shows that the four-loop expression eliminates virtually all of the residual scale dependence still evident in the three-loop rate in the region $M_{t} \geq \mu \geq 30 \mathrm{GeV}$. The four-loop rate is observed to have a local minimum at $\mu=43.5 \mathrm{GeV}$ and a weak local maximum at $\mu=89.5 \mathrm{GeV}$. The values of $S[x(\mu), L(\mu), T]$ at both of these points of minimal-sensitivity [13] differ by only $0.2 \%$, indicative of the flatness of $S$ between these two points. In Table 3 , values for $S[x(\mu), L(\mu), T]$ as well as the term-by-term series $=x^{2}\left[1+R_{1} x+R_{2} x^{2}+R_{3} x^{3}\right]$ within $S$ are displayed for a variety of $\mu$-values of interest. The table displays the relative size of successive terms $R_{n} x^{n}$ at the minimalsensitivity points $\mu=43.5$ and $89.5 \mathrm{GeV}$ in addition to the points $\mu=140 \mathrm{GeV}\left(=M_{H}\right)$ and $\mu=175 \mathrm{GeV}\left(\cong M_{t}\right)$. The relative magnitude of the four-loop term $R_{3} x^{3}$ is seen to be less than $1 \%$ of the leading term in the series (unity) over the entire region between the minimal-sensitivity values of $\mu$.

Of particular interest in this range are those values of $\mu[47.0 \mathrm{GeV}$ and $73.5 \mathrm{GeV}]$ at which the four-loop term $R_{3} x^{3}$ effectively vanishes. These values correspond to the two points in Figure 1 at which the 3-loop and 4-loop curves cross. The usual approach towards extracting information from an asymptotic series $\sum_{n=0} R_{n} x^{n}\left(R_{0}=1\right)$ is to sum only that series' decreasing terms, i.e. to evaluate $\sum_{n=0}^{n^{\prime}} R_{n} x^{n}$ by choosing $n^{\prime}$ such that $\left|R_{n^{\prime}} x^{n^{\prime}}\right|$ is a minimum. By choosing $\mu$ so as to have $R_{3}(\mu)$ vanish, one can then argue (for this choice of $\mu$ ) that $n^{\prime}=3$, suggesting that such a value for $\mu$ is optimal for estimating the series from its known terms. Of course, such an 
interpretation rests on the assumed increase of terms $\left|R_{n} x^{n}\right|$ subsequent to $n^{\prime}=3$; all we can really be certain of is that $\left|R_{4} x^{4}\right|>\left|R_{3} x^{3}\right|(=0)$ at such a value of $\mu$. Nevertheless, Table 3 shows that both values of $\mu$ for which $R_{3} \rightarrow 0$ lie between the two minimal-sensitivity points $[\mu=43.5$ and $89.5 \mathrm{GeV}]$, and that the $\mu$-sensitive factor $S[x(\mu), L(\mu), T]$ within the rate (2.6) varies by less than $0.2 \%$ over this entire region. Over the full range of $\mu$ values displayed in Table $3(43.5 \mathrm{GeV}<\mu<175 \mathrm{GeV})$, the four-loop series term $\left(R_{3} x^{3}\right)$ varies between 0 and $5 \%$ of the leading one-loop order term (unity). Surprisingly, however, the rate $S[x(\mu), L(\mu), T]$ displays a relative spread of values $\Delta S / S \leq 0.4 \%$ over the same range of $\mu$, indicative of a substantial reduction in residual scale dependence.

In Figures 2 and 3 we exhibit the residual scale dependence of $S[x(\mu), L(\mu), T]$ to three and four-loop order for Higgs masses of $100 \mathrm{GeV}$ and $175 \mathrm{GeV}$, respectively. These figures show the same reduction in scale dependence evident in Figure 1 when $M_{H}=140 \mathrm{GeV}$. For the case of $M_{H}=100 \mathrm{GeV}$, for example, Table 4 shows that the 4-loop term $R_{3} x^{3}$ varies between zero and $10 \%$ of the leading-order 1-loop term between the local minimum at $\mu=29 \mathrm{GeV}$ and $\mu \cong M_{t}$. Over this same range of $\mu$, the four-loop values of $S[x(\mu), L(\mu), T]$ remain within $1 \%$ of each other. For $M_{H}=175 \mathrm{GeV}$, the four-loop term is between zero and $3 \%$ of the leading-order term between the local minimum at $\mu=48.5 \mathrm{GeV}$ and $\mu \cong M_{t}$, whereas the full four-loop expression for $S[x(\mu), L(\mu), T]$ exhibits a relative spread of only $0.1 \%$ [Table 5$]$.

Such scale independence is, of course, a reflection of the $R G$-invariance $(2.8)$ of $S[x(\mu), L(\mu), T]$, which has been utilized explicitly to obtain the coefficients $c_{1-3}$. Nevertheless, the coefficient $c_{0}$, which is not perturbatively accessible via (2.8) but is obtainable (at present) only by Padé approximant methods, appears to be precisely what is required to eliminate virtually all residual $\mu$-dependence in the rate arising from truncation. For example, if $M_{H}=100 \mathrm{GeV}$, the factor $c_{0} x^{3}(\mu)$ in isolation contributes more than $10 \%$ of the leading order contribution (unity) for $\mu \lesssim M_{t}$. Moreover, this contribution increases as $\mu$ decreases. Nevertheless, the particular choice $c_{0}=2453$ appears to ensure that the overall spread in $S$ remains within $1 \%$, despite the potentially large contribution to this spread from $c_{0} x^{3}(\mu)$. Evidently the $\mu$ dependence of this term serves to cancel the residual $\mu$-dependence of the remaining terms in the series. 1

This near cancellation of residual scale-dependence makes possible a set of credible predictions for the rate (2.6). In the fourth column of Table 6 we have tabulated the $H \rightarrow g g$ decay rates for $M_{H}=100,125,140,150$, and $175 \mathrm{GeV}$ in the $m_{b} \rightarrow 0, M_{H}^{2}<<4 M_{t}^{2}$ limit. The largest source of uncertainty for these predictions is in the value for $\alpha_{s}\left(M_{z}\right)=(0.119 \pm 0.002$ [12] $)$, which should lead to $4 \%$ uncertainty in the rates presented in Table 6 .

Fermion mass effects (i.e. the departures from the $m_{b} \rightarrow 0, M_{H}^{2}<<4 M_{t}^{2}$ assumptions implicit in the derivation [1] of the $H \rightarrow g g$ rate) can be accommodated in leading order by replacing the factor of unity in (3.1) with the

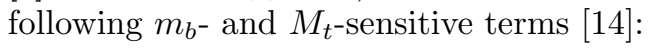

$$
\begin{aligned}
& 1 \rightarrow 1+\delta_{m}=\frac{9}{16}\left[\left(A_{t}+\operatorname{Re} A_{b}\right)^{2}+\left(\operatorname{Im} A_{b}\right)^{2}\right], \\
& A_{t}=2\left[\tau_{t}+\left(\tau_{t}-1\right)\left(\sin ^{-1}\left(\sqrt{\tau_{t}}\right)\right)^{2}\right] / \tau_{t}^{2}, \quad \tau_{t} \equiv M_{H}^{2} / 4 M_{t}^{2}, \\
& A_{b}=2\left[\tau_{b}+\left(\tau_{b}-1\right) f\left(\tau_{b}\right)\right] / \tau_{b}^{2}, \quad \tau_{b} \equiv M_{H}^{2} / 4 m_{b}^{2}, \\
& f(\tau) \equiv-\frac{1}{4}\left[\ln \left(\frac{1+\sqrt{1-1 / \tau}}{1-\sqrt{1-1 / \tau}}\right)-i \pi\right]^{2} .
\end{aligned}
$$

The right hand side of (4.1) is easily seen to approach unity when $m_{b} \rightarrow 0, \quad M_{H}^{2} / 4 M_{t}^{2} \rightarrow 0$ :

$$
\lim _{\tau_{t} \rightarrow 0} A_{t}=\frac{4}{3} ; \lim _{\tau_{b} \rightarrow \infty} A_{b}=0 .
$$

The leading mass correction $\delta_{m}$, as defined in (4.1), is tabulated for various Higgs boson masses (with PDG [12] fermion-mass values $m_{b}=4.2 \mathrm{GeV}, M_{t}=175.6 \mathrm{GeV}$ ) in the fifth column of Table 6 . This lowest-order fermion mass correction can be incorporated into the rate (2.6) by its inclusion into the series (3.1)

$$
S=x^{2}\left[1+R_{1} x+R_{2} x^{2}+R_{3} x^{3}+\delta_{m}\right] .
$$

\footnotetext{
${ }^{1}$ The reduction of scale-dependence via Padé approximant methods is discussed in detail by Gardi $[5$.
} 
The sixth column of Table 6 tabulates the $H \rightarrow g g$ rate with this correction included.

Because $\delta_{m}$ is a leading order correction, there is genuine ambiguity as to whether "physical" or running masses should be incorporated into this correction. The former choice yields a manifestly scale-dependent contribution $\Delta S=\delta_{m}^{p} x^{2}(\mu)$ to the rate $S$. One could argue for the incorporation of running masses $m_{b}(\mu), m_{t}(\mu)$ in $(4.2)$ and (4.3); i.e., $\Delta S=\delta_{m}(\mu) x^{2}(\mu)$, with $\delta_{m}(\mu)$ calculated via (4.1-4) with $\tau_{t}=M_{H}^{2} / 4 m_{t}^{2}(\mu)$ and $\tau_{b}=M_{H}^{2} / 4 m_{b}^{2}(\mu)$, and with $\mu$ identified consistently with the minimal-sensitivity scale tabulated in Column 2 of Table 6 . In Column 7 of Table 6 we have tabulated $\delta_{m}(\mu)$ utilizing 6 -active-flavour running masses referenced to $m_{t}\left(m_{t}\right)=175.6 \mathrm{GeV}$, $m_{b}\left(m_{b}\right)=4.2 \mathrm{GeV}$, and in Column 8 we have listed the corresponding $H \rightarrow g g$ decay rates. Theoretical uncertainty associated with the masses utilized in the leading correction-factor $\delta_{m}$ is reflected in the differing rates of Column 6 and Column 8. This discrepancy is seen to be at most a $2 \%$ effect.

The leading order mass-correction factor $\delta_{m}$ in (4.6) is seen from a comparison of Table 6 to Tables 3-5 to be generally smaller than the three-loop term $R_{2} x^{2}$ in (4.6), but somewhat larger than the four-loop term $R_{3} x^{3}$ also appearing in (4.6) $\left[\delta_{m}\right.$ is smaller than $R_{3} x^{3}$ when $\mu=M_{t}$, as in Table 2]. Since the next order of fermion mass corrections is suppressed by an additional power of $\alpha_{s}$, we anticipate such next-to-leading-order fermion mass corrections to be well within $1 \%$ of the total rate. In any case, both leading-order mass corrections $\delta_{m}$ and the four-loop contribution $R_{3} x^{3}$ we obtain are sufficiently small for credible estimates of the $H \rightarrow g g$ rate on the basis of the terms in (4.6). It is also worth noting from Table 6 that $\delta_{m}^{p}$ itself is only $1 \%$ of the leading-order one loop term [normalized to unity in (4.6)] when $M_{H}=140-150 \mathrm{GeV}$, and that $\delta_{m}(\mu)$ is comparably small when $M_{H}=150 \mathrm{GeV}$. Hence, the rates tabulated in Table 6 are optimally valid for Higgs masses in the $140-150 \mathrm{GeV}$ range.

\section{The $H \rightarrow b \bar{b}$ rate}

The leading QCD scalar-current correlation function contributions to the Higgs $\rightarrow b \bar{b}$ decay rate are known from explicit calculation to $\mathcal{O}\left(\alpha_{s}^{3}\right)$, with secondary $\mathcal{O}\left(m_{b}^{2} / M_{H}^{2}\right)$ power corrections known to $\mathcal{O}\left(\alpha_{s}^{2}\right)$, as given in $[7]$ :

$$
\begin{aligned}
\Gamma(H \rightarrow b \bar{b})=\frac{3 G_{F}}{4 \sqrt{2} \pi} M_{H} m_{b}^{2}\left(M_{H}\right)\left[\Pi_{\text {scalar }}\left(\mu^{2}=s=M_{H}^{2}\right)\right. \\
\left.\quad+\frac{m_{b}^{2}\left(M_{H}\right)}{M_{H}^{2}}\left(-6-40 \frac{\alpha_{s}\left(M_{H}\right)}{\pi}-87.72\left(\frac{\alpha_{s}\left(M_{H}\right)}{\pi}\right)^{2}\right)\right] .
\end{aligned}
$$

The factor $\Pi_{\text {scalar }}$ in (5.1) is the imaginary part of the QCD correlation function for the quark-antiquark scalar current normalized to unity in leading order [7]:

$$
\begin{aligned}
\Pi_{\text {scalar }}[\mu, s, x(\mu)]=1 & +\left(\frac{17}{3}+2 \ln \mu^{2} / s\right) x(\mu) \\
& +\left(29.1467+\frac{263}{9} \ln \left(\frac{\mu^{2}}{s}\right)+\frac{47}{12} \ln ^{2}\left(\frac{\mu^{2}}{s}\right)\right) x^{2}(\mu) \\
& +\left(41.7576+238.381 \ln \left(\frac{\mu^{2}}{s}\right)+94.6759 \ln ^{2}\left(\frac{\mu^{2}}{s}\right)+7.61574 \ln ^{3}\left(\frac{\mu^{2}}{s}\right)\right) x^{3}(\mu) \\
& +\left(d_{0}+d_{1} \ln \left(\frac{\mu^{2}}{s}\right)+d_{2} \ln ^{2}\left(\frac{\mu^{2}}{s}\right)+d_{3} \ln ^{3}\left(\frac{\mu^{2}}{s}\right)+d_{4} \ln ^{4}\left(\frac{\mu^{2}}{s}\right)\right) x^{4}(\mu) \\
& +\ldots
\end{aligned}
$$

where $x(\mu)=\left[\alpha_{s}(\mu)\right]_{n_{f}=5} / \pi$, corresponding to five active flavours.

In ref.[2], a detailed asymptotic Padé-approximant procedure is presented for estimating the coefficients $d_{0-4}$ in (5.2). The methodology is virtually identical to that of Section 3 above, except that five moments are now calculated for $R_{4}[w]$, the coefficient of $x^{4}(\mu)$, based upon the asymptotic error formula prediction [11]

$$
R_{4}=\frac{R_{3}^{2}\left[R_{2}^{3}+R_{1} R_{2} R_{3}-2 R_{1}^{3} R_{3}\right]}{R_{2}\left[2 R_{2}^{3}-R_{1}^{3} R_{3}-R_{1}^{2} R_{2}^{2}\right]} .
$$


The results, as tabulated in Table 3 of ref. [2], are

$$
d_{0}=64.2, d_{1}=745, d_{2}=1180, d_{3}=253, d_{4}=15.4 .
$$

The factors $d_{1-4}$ may be obtained directly from RG-invariance of the physical rate

$$
\mu^{2} \frac{d}{d \mu^{2}}\left[m_{b}^{2}(\mu) \Pi_{\text {scalar }}[\mu, s, x(\mu)]\right]=0,
$$

leading to the following values (also tabulated) in [2]):

$$
d_{1}=791.52, d_{2}=1114.7, d_{3}=260.06, d_{4}=14.755 .
$$

The strong agreement between (5.6) and (5.4) suggests that the estimate for $d_{0}$ in (5.4) is a credible one. It is interesting to note that a much more naive Padé approach for estimating $d_{0}$, in which (5.3) was applied directly to $\Pi_{\text {scalar }}\left(\mu^{2}=s=M_{H}^{2}\right)$, yielded a value for $d_{0}(=67.25$ [1] $)$ surprisingly consistent with the estimate in (5.4). Only the latter estimate exhibits sensitivity to the logarithmic terms in $\Pi_{\text {scalar }}$, which all vanish when $\mu^{2}=s$.

If we utilize the estimate for $d_{0}$ quoted in (5.4) within the $H \rightarrow b \bar{b}$ rate, we find that the correlation-function factor within $(5.1)$ is given to $\mathcal{O}\left(\alpha_{s}^{4}\right)$ by

$$
\Pi_{\text {scalar }}\left(\mu^{2}=s=M_{H}^{2}\right)=1+\frac{17}{3} x\left(M_{H}\right)+29.1467 x^{2}\left(M_{H}\right)+41.7576 x^{3}\left(M_{H}\right)+\underline{64} x^{4}\left(M_{H}\right) .
$$

The underlined coefficient is, of course, $d_{0}$ as estimated in [2] via asymptotic Padé-approximant methods.

There is genuine value in having an estimate of this term, as it is generally larger than the $\mathcal{O}\left(\alpha_{s}^{2} m_{b}^{2} / M_{H}^{2}\right)$ final term in (5.1). For example, suppose that $M_{H}=130 \mathrm{GeV}, m_{b}(130 \mathrm{GeV})=2.7 \mathrm{GeV}$ and $x(130 \mathrm{GeV})=0.114 / \pi$ (these values are consistent with those employed in Table 1 of ref. [7]). The relative magnitudes of the correlator contributions 95.5) to the $H \rightarrow b \bar{b}$ rate are seen to be

$$
\Pi_{\text {scalar }}\left(\mu^{2}=s=(130 \mathrm{GeV})^{2}\right)=1+0.2056+0.0384+0.0020+\underline{0.00011} .
$$

All but the underlined Padé-estimate term are tabulated in Table 1 of ref. [7]. Comparison to the power-suppressed terms in (5.1),

$$
\frac{m_{b}^{2}\left(M_{H}\right)}{M_{H}^{2}}\left(-6-40 x\left(M_{H}\right)-87.72 x^{2}\left(M_{H}\right)\right) \underset{M_{H}=130 \mathrm{GeV}}{\longrightarrow}-0.0026-0.00062-0.00005,
$$

(also tabulated in [7]) reveals that the $\mathcal{O}\left(\alpha_{s}^{4}\right)$ term in (5.8) is double the magnitude of the $\mathcal{O}\left(\alpha_{s}^{2}\right)$ term in $(5.9)$. Hence the Padé-estimated $\mathcal{O}\left(\alpha_{s}^{4}\right)$ term in (5.7) enables one to utilize the full precision available in the known power-suppressed contributions to (5.1). However, one cannot anticipate such precision experimentally for many years to come.

\section{Summary}

In the preceding sections, we have demonstrated how asymptotic Padé-approximant methods may be utilized to estimate the unknown $\mathcal{O}\left(\alpha_{s}^{5}\right)$ coefficients $\left\{c_{0}, c_{1}, c_{2}, c_{3}\right\}$ within the $H \rightarrow 2 g$ decay rate (2.7). Such estimates for $\left\{c_{1}, c_{2}, c_{3}\right\}$ are seen (Table 1 ) to be within a few percent of the true values for these coefficients, which can be extracted via renormalization-group methods. This accuracy supports corresponding asymptotic Padé-approximant estimates of the renormalization-group-inaccessible coefficients $c_{0}$ presented in Table 1 . Moreover, the inclusion of $\mathcal{O}\left(\alpha_{s}^{5}\right)$ terms within $(2.7)$ is seen to virtually eliminate the scale-parameter dependence of the rate over an astonishingly large range of the scale-parameter $\mu$, typically $0.3 M_{H} \lesssim \mu \lesssim M_{t}$. Inclusion of estimated $\mathcal{O}\left(\alpha_{s}^{5}\right)$ corrections in conjunction with leading-order fermion-mass corrections to the rate (Table 6) are seen to reduce the perturbative uncertainty in the $H \rightarrow 2 g$ decay rate from $\mathcal{O}(20 \%)$ to $\mathcal{O}(2 \%)$. Four-loop corrections to the $H \rightarrow b \bar{b}$ decay mode are also presented, which are seen to reduce the very small perturbative uncertainty of this dominant hadronic mode by an additional order of magnitude. 


\section{Acknowledgement}

We are grateful for support from the Natural Sciences and Engineering Research Council of Canada.

\section{References}

[1] K. G. Chetyrkin, B. A. Kniehl, and M. Steinhauser, Phys. Rev. Lett. 79 (1997) 353.

[2] F. Chishtie, V. Elias, and T.G. Steele, Phys. Rev. D 59 (1999) 105013.

[3] F. A. Chishtie, V. Elias, and T.G. Steele, J. Phys. G 26 (2000) 93.

[4] J. Ellis, E. Gardi, M. Karliner and M. A. Samuel, Phys. Lett. B 366 (1996) 268 and Phys. Rev. D 54 (1996).

[5] E. Gardi, Phys. Rev. D 56 (1997) 68.

[6] M.R. Ahmady, F. A. Chishtie, V. Elias, and T.G. Steele, Phys. Lett. B (to appear: hep-ph/9910551).

[7] K. G. Chetyrkin, Phys. Lett. B 390 (1997) 309.

[8] T. van Ritbergen, Phys. Lett. B 454 (1999) 353.

[9] S. A. Larin, T. van Ritbergen, and J. A. M. Vermaseren, Nucl. Phys. B 438 (1995) 278.

[10] J. Ellis, I. Jack, D.R.T. Jones, M. Karliner, and M. Samuel, Phys. Rev D 57 (1998) 2665.

J. Ellis, M. Karliner, and M. A. Samuel, Phys. Lett. B 400 (1997) 176.

[11] V. Elias, T.G. Steele, F. Chishtie, R. Migneron, and K. Sprague, Phys. Rev. D 58 (1998) 116007.

[12] C. Caso et. al. [Particle Data Group], Eur. Phys. J. C 3 (1998) 1.

[13] P.M. Stevenson, Phys. Rev. D 23 (1981) 2916.

[14] M. Spira, A. Djouadi, D. Graudenz and P.M. Zerwas, Nucl. Phys. B 453 (1995) 17. 


\begin{tabular}{ccccc}
$M_{H}(\mathrm{GeV})$ & $\delta c_{1} / c_{1}^{R G}$ & $\delta c_{2} / c_{2}^{R G}$ & $\delta c_{3} / c_{3}^{R G}$ & $c_{0}$ \\
\hline 100 & -0.010 & -0.019 & -0.054 & 2453 \\
125 & -0.009 & -0.013 & -0.059 & 1646 \\
140 & -0.009 & -0.009 & -0.062 & 1306 \\
150 & -0.009 & -0.007 & -0.068 & 1120 \\
175 & -0.012 & -0.0004 & -0.070 & 763
\end{tabular}

Table 1: The final column tabulates asymptotic Padé-approximant predictions for the four loop nonlogarithmic coefficient $c_{0}$ within (2.7), as obtained via moments of $c_{0}-c_{1} l n w+c_{2} l n^{2} w-c_{3} l^{3} w$, for Higgs masses between $100 \mathrm{GeV}$ and $M_{t}$. Relative errors between predictions and true values of $c_{1}, c_{2}$ and $c_{3}$, as determined from RG invariance, are tabulated in the second, third and fourth column: $\delta c_{i}=\left(c_{i}^{\text {predicted }}-c_{i}^{R G}\right) / c_{i}^{R G}$.

\begin{tabular}{c|c}
$M_{H}(\mathrm{GeV})$ & $S\left[x\left(M_{t}\right), L\left(M_{t}\right), T\right] / x^{2}=1+a_{0} x+b_{0} x^{2}+c_{0} x^{3}$ \\
\hline 100 & $1+0.7728+0.3333+\underline{0.1014}$ \\
125 & $1+0.7133+0.2675+\underline{0.0679}$ \\
140 & $1+0.6831+0.2361+\underline{0.0538}$ \\
150 & $1+0.6647+0.2177+\underline{0.0461}$ \\
175 & $1+0.6237+0.1783+\underline{0.0312}$
\end{tabular}

Table 2: The perturbative series $S / x^{2}$ within the $H \rightarrow 2 g$ decay rate $(2.7)$, evaluated at $\mu=m_{t}(\mu)=175.6 \mathrm{GeV}$. $x(175.6 \mathrm{GeV})=0.10915 / \pi$, consistent with $x\left(M_{z}\right)=\alpha_{s}\left(M_{z}\right) / \pi=0.119 / \pi$. The final underlined term of the series is obtained from the $c_{0}$ estimates in Table 1.

\begin{tabular}{c|c|c|c|c}
$\mu(\mathrm{GeV})$ & $\alpha_{s}(\mu)$ & $m_{t}(\mu)(\mathrm{GeV})$ & $1+R_{1} x+R_{2} x^{2}+R_{3} x^{3}$ & $S[x(\mu), L(\mu), T]$ \\
\hline 43.5 & 0.1325 & 198.2 & $1+0.3811-0.0395+0.0023$ & 0.002390 \\
47.0 & 0.1309 & 196.8 & $1+0.4014-0.0251-0.0001$ & 0.002390 \\
73.5 & 0.1226 & 188.8 & $1+0.5094+0.0614+0.0000$ & 0.002393 \\
89.5 & 0.1193 & 185.6 & $1+0.5525+0.1007+0.0068$ & 0.002394 \\
140 & 0.1124 & 178.8 & $1+0.6419+0.1907+0.0344$ & 0.002390 \\
175 & 0.1092 & 175.6 & $1+0.6825+0.2354+0.0534$ & 0.002384
\end{tabular}

Table 3: The four-loop expression for the scale-dependent factor $S[x(\mu), L(\mu), T]$ for $M_{H}=140 \mathrm{GeV}$ within the Higgs $\rightarrow$ gluon-gluon decay rate (2.6) for five different values of $\mu$. Weak extrema within the rate (Fig. 1) occur at $\mu=43.5$ and $89.5 \mathrm{GeV}$. Also displayed are $\mu=M_{H}, \mu \approx M_{t}$, and two values of $\mu(47.0 \mathrm{GeV}$ and $73.5 \mathrm{GeV})$ at which the four-loop contribution to $S$ is almost zero. The values of $S$ over this entire range of $\mu$ are seen to be remarkably static, as discussed in the text.

\begin{tabular}{c|c|c|c|c}
$\mu(\mathrm{GeV})$ & $\alpha_{s}(\mu)$ & $m_{t}(\mu)(\mathrm{GeV})$ & $1+R_{1} x+R_{2} x^{2}+R_{3} x^{3}$ & $S[x(\mu), L(\mu), T]$ \\
\hline 29.0 & 0.1413 & 206.5 & $1+0.3819-0.0546+0.0033$ & 0.002691 \\
31.0 & 0.1397 & 205.1 & $1+0.4007-0.0410+0.0002$ & 0.002691 \\
54.0 & 0.1282 & 194.2 & $1+0.5418+0.0775+0.0001$ & 0.002698 \\
65.5 & 0.1247 & 190.8 & $1+0.5853+0.1198+0.0089$ & 0.002699 \\
100 & 0.1175 & 183.8 & $1+0.6722+0.2125+0.0402$ & 0.002693 \\
175 & 0.1092 & 175.6 & $1+0.7722+0.3326+0.1010$ & 0.002667
\end{tabular}

Table 4: The scale-dependence of the four-loop expression for $S[x(\mu), L(\mu), T]$ is displayed, as in Table 3, for the case $M_{H}=100 \mathrm{GeV}$. Weak extrema in Figure 2 occur at $\mu=29.0$ and $65.5 \mathrm{GeV}$. The four-loop contribution is seen to nearly vanish at $\mu=31.0$ and $54.0 \mathrm{GeV}$. 


\begin{tabular}{c|c|c|c|c}
$\mu(\mathrm{GeV})$ & $\alpha_{s}(\mu)$ & $m_{t}(\mu)(\mathrm{GeV})$ & $1+R_{1} x+R_{2} x^{2}+R_{3} x^{3}$ & $S[x(\mu), L(\mu), T]$ \\
\hline 48.5 & 0.1303 & 196.2 & $1+0.3385-0.0584+0.0081$ & 0.002216 \\
61.0 & 0.1260 & 192.0 & $1+0.3976-0.0190-0.0001$ & 0.002216 \\
91.0 & 0.1190 & 185.3 & $1+0.4912+0.0537+0.0000$ & 0.002218 \\
111 & 0.1159 & 182.2 & $1+0.5338+0.0910+0.0058$ & 0.002218 \\
175 & 0.1092 & 175.6 & $1+0.6230+0.1777+0.0309$ & 0.002215
\end{tabular}

Table 5: The scale-dependence of the four-loop expression for $S[x(\mu), L(\mu), T]$ is displayed, as in Table 3, for the case $M_{H}=175 \mathrm{GeV}$. Weak extrema in Figure 3 occur at $\mu=48.5$ and $111.0 \mathrm{GeV}$. The four-loop term is seen to nearly vanish at $\mu=61.0$ and $91.0 \mathrm{GeV}$. The relative spread in the rate $S$ is seen to be only of order $0.1 \%$ for values of $\mu$ between 48.5 and $175 \mathrm{GeV}$.

\begin{tabular}{cccccccc}
$M_{H}$ & $\mu$ & $S$ & $\Gamma_{H \rightarrow g g}^{\delta_{m}=0}$ & $\delta_{m}^{p}$ & $\Gamma_{H \rightarrow g g}^{\delta_{m}^{p}}$ & $\delta_{m}(\mu)$ & $\Gamma_{H \rightarrow g g}^{\delta_{m}(\mu)}$ \\
\hline 100 & 65.5 & 0.00270 & $1.97 \cdot 10^{-4}$ & -0.085 & $1.87 \cdot 10^{-4}$ & -0.059 & $1.90 \cdot 10^{-4}$ \\
125 & 80.5 & 0.00249 & $3.55 \cdot 10^{-4}$ & -0.040 & $3.47 \cdot 10^{-4}$ & -0.015 & $3.52 \cdot 10^{-4}$ \\
140 & 89.5 & 0.00239 & $4.79 \cdot 10^{-4}$ & -0.012 & $4.76 \cdot 10^{-4}$ & +0.011 & $4.82 \cdot 10^{-4}$ \\
150 & 95.5 & 0.00234 & $5.75 \cdot 10^{-4}$ & +0.0076 & $5.78 \cdot 10^{-4}$ & +0.023 & $5.83 \cdot 10^{-4}$ \\
175 & 111 & 0.00222 & $8.67 \cdot 10^{-4}$ & +0.060 & $8.99 \cdot 10^{-4}$ & +0.077 & $9.08 \cdot 10^{-4}$
\end{tabular}

Table 6: The four-loop order $H \rightarrow g g$ decay rate (2.6) is tabulated for various choices of $M_{H}$. The second column lists the minimal-sensitivity values of $\mu(d S / d \mu=0)$ closest to $M_{H}$. The third column is the value of $S[x(\mu), L(\mu), T]$ evaluated at this choice for $\mu$, as indicated in the previous three tables. The fourth column is the rate in the $m_{b} \rightarrow 0, M_{H}^{2}<<4 M_{t}^{2}$ limit. The fifth column tabulates the magnitudes of the leading fermion mass correction to $S / x^{2}$, as discussed in the text, using "physical" (p) fermion masses $M_{t}=175.6 \mathrm{GeV}, m_{b}=4.2 \mathrm{GeV}$.The sixth column tabulates the $H \rightarrow g g$ rate incorporating this mass correction. The seventh column lists the leading fermion mass correction to $S / x^{2}$ utilizing running fermion mass values $m_{b}(\mu), m_{t}(\mu)$ at the Column 2 values of $\mu$, and the final column tabulates the $H \rightarrow g g$ rate incorporating this fermion-mass correction. All masses and decay rates are in $\mathrm{GeV}$ units. 
Fig.1

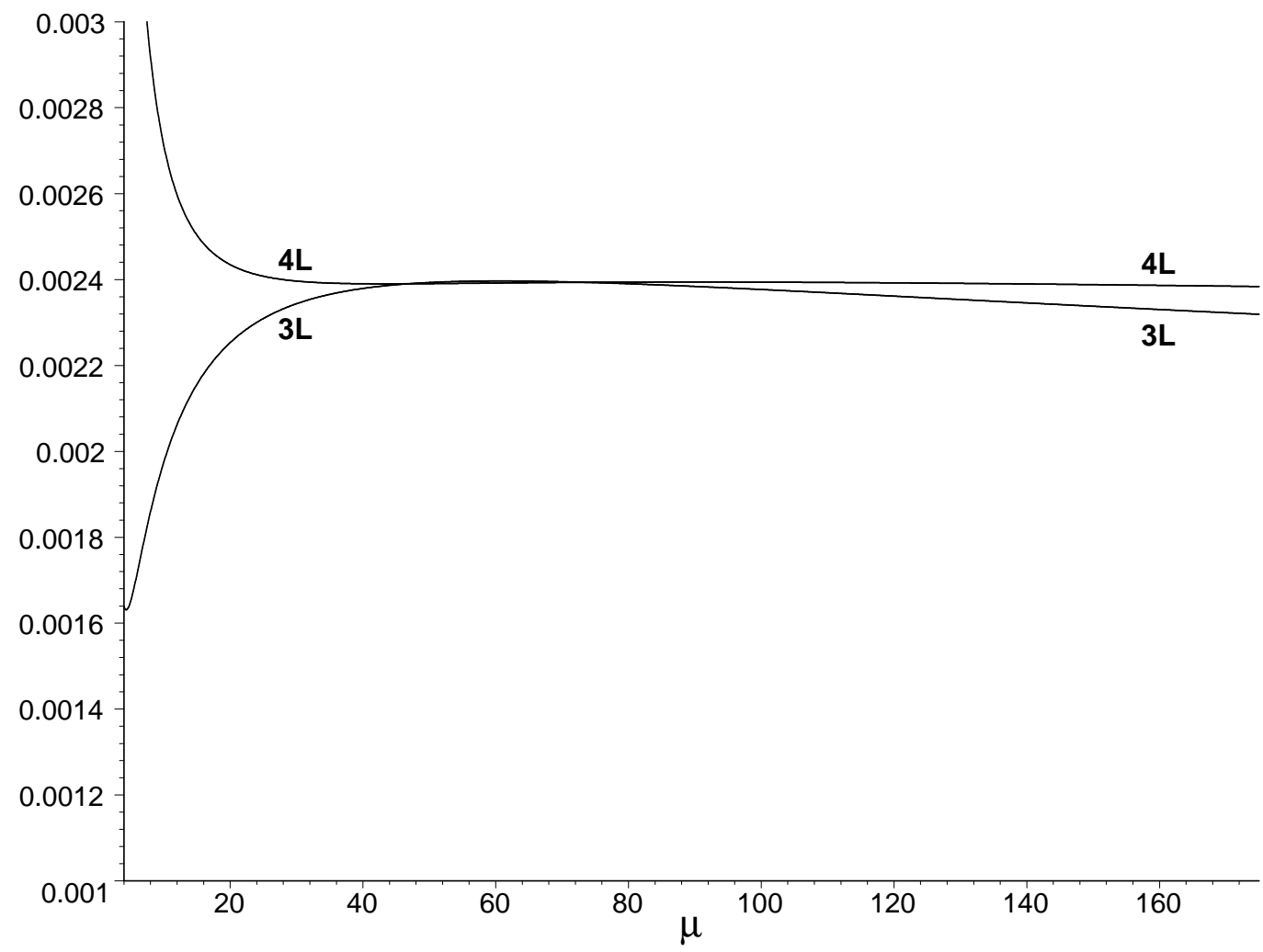

Figure 1: Comparison of the residual renormalization scale- $(\mu-)$ dependence of the three-loop (3L) and fourloop (4L) expression for the $H \rightarrow g g$ decay rate of a Higgs boson with mass $M_{H}=140 \mathrm{GeV}$. The $y$-axis numbers correspond to the dimensionless scale-dependent factor $S[x(\mu), L(\mu), T]$ within the rate $(2.6)$, and the $x$-axis is the scale $\mu$ in $\mathrm{GeV}$. The logarithmic coefficients of $4 \mathrm{~L}$-contributions are extracted via renormalization-group equation methods. The $4 \mathrm{~L}$ non-logarithmic coefficient is obtained via asymptotic Padé approximant methods. The $3 \mathrm{~L}$ and $4 \mathrm{~L}$ curves nearly coincide in the region $44 \mathrm{GeV} \lesssim \mu \lesssim 80 \mathrm{GeV}$, indicating a very small $4 \mathrm{~L}$ contribution over this range of $\mu$. 
Fig.2

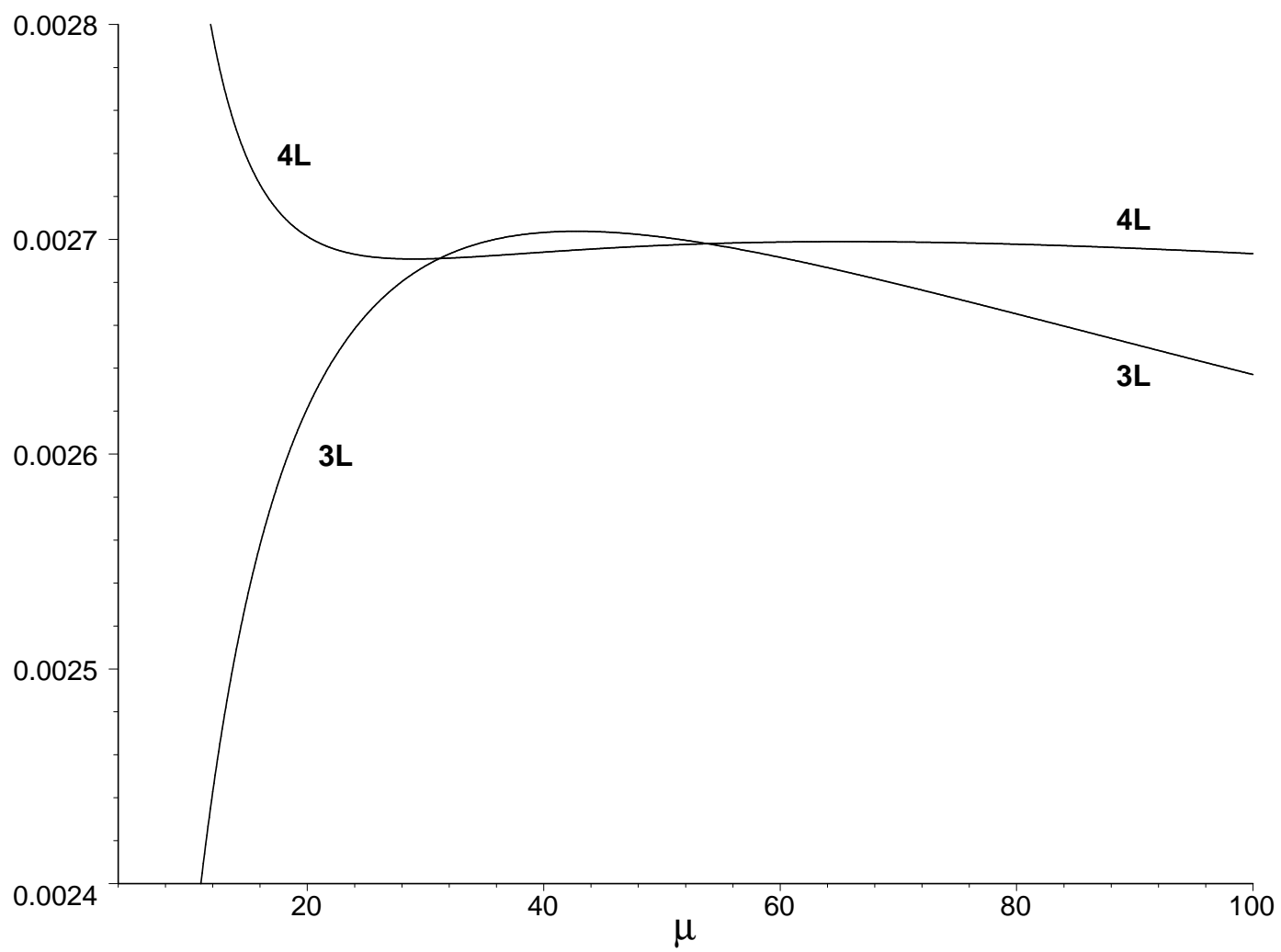

Figure 2: A comparison of the $\mu$-dependence of three-loop and four-loop expressions for the $H \rightarrow g g$ rate is plotted, as in Figure 1, but now for $M_{H}=100 \mathrm{GeV}$. The $y$-axis numbers correspond to the dimensionless scale-dependent factor $S[x(\mu), L(\mu), T]$ within the rate $(2.6)$, and the $x$-axis is the scale $\mu$ in GeV. The curves cross at values of $\mu$ for which the (estimated) four-loop contribution to the rate is zero. 
Fig.3

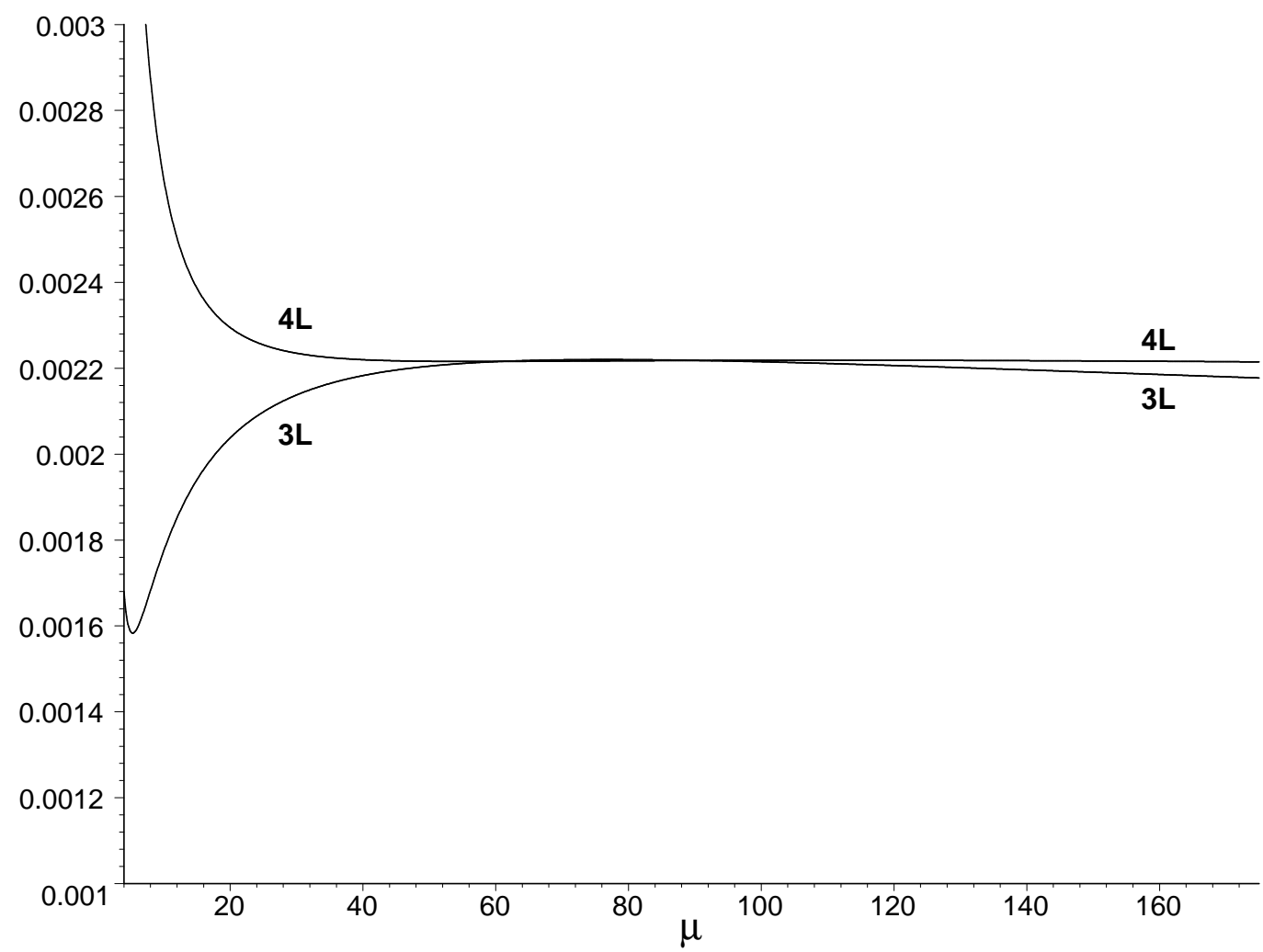

Figure 3: A comparison of the $\mu$-dependence of three-loop and four-loop expressions for the $H \rightarrow g g$ rate (as in Figs. 1 and 2), for $M_{H}=175 \mathrm{GeV}$. The $y$-axis numbers correspond to the dimensionless scale-dependent factor $S[x(\mu), L(\mu), T]$ within the rate $(2.6)$, and the $x$-axis is the scale $\mu$ in $\mathrm{GeV}$. 